\title{
Leitlinien chronischer Rückenschmerz
}

— Das Institut für Qualität und Wirtschaftlichkeit im Gesundheitswesen (IQWiG) untersuchte sechs Leitlinien zum chronischen Rückenschmerz, um Empfehlungen für ein mögliches neues Disease-ManagementProgramm (DMP) Chronischer Rückenschmerz zu identifizieren. Dabei wurden laut IQWiG Aspekte wie Diagnostik, Therapie und Rückfallprophylaxe ausgewertet. In seinem Abschlussbericht kam das IQWiG zu dem Schluss, dass die analysierten Leitli- nien alle wichtigen Versorgungsaspekte abdecken.

Für Empfehlungen bezüglich der medikamentösen und invasiven Therapie herrschte größtenteils Konsistenz. Einstimmigkeit bestand vor allem bei nicht medikamentösen Behandlungen wie Massagen, manueller Therapie, Bewegung, Physiotherapie sowie Yoga. Gerade für diese Anwendungen zeigten allerdings nicht alle Leitlinien eine ausreichende Evidenz.

(red)

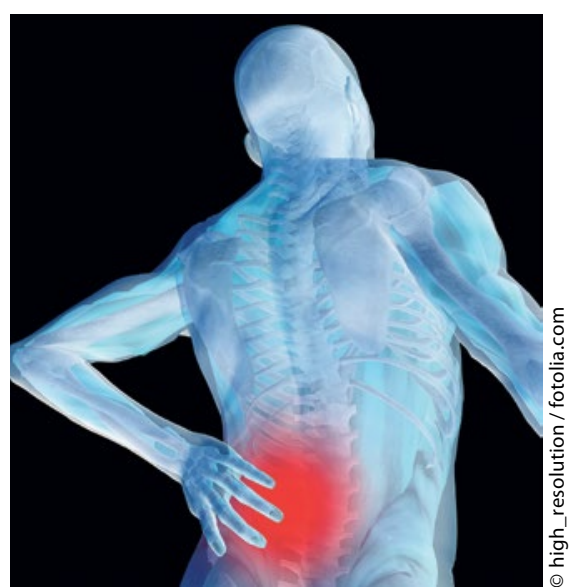

und -psychologen, Physiotherapeuten und Fachärzten der Neurochirurgie und Orthopädie individuell auf den Patienten abgestimmt, um die Schmerzen zu lindern und die Funktionsfähigkeit des Patienten wieder herzustellen, erläutert Bingel.

Für eine Behandlung im Rückenschmerzzentrum benötigen die Patienten eine Überweisung durch einen niedergelassenen Haus- oder Facharzt mit der Indikation „Rückenschmerzen“. Die Behandlung kann ambulant, tagesklinisch oder stationär erfolgen.

(red)

Urteil

\section{Kein Recht auf tödliche Arznei-Dosis}

— Bei einem Suizidwunsch muss der Staat nicht den Zugang zur tödlichen Arzneidosis erlauben. Dies verstößt weder gegen das Grundgesetz noch gegen die Europäische Menschenrechtskonvention, wie das Verwaltungsgericht Köln entschied.

Die 78 und 71 Jahre alten Kläger hatten beim Bundesinstitut für Arzneimittel und Medizinprodukte (BfArM) erfolglos den Kauf einer tödlichen Dosis Natrium-Pentobarbital beantragt. Das Paar gab als Begründung an, Suizid begehen zu wollen. Sie seien zwar nicht schwer krank, würden aber das Nachlassen ihrer körperlichen und geistigen Kräfte merken. Um sich und ihren Angehörigen einen jahrelangen Verfall und qualvollen Tod zu er- sparen, hätten sie ein Recht auf ein „selbstbestimmtes Ableben mit einem Mittel ihrer Wahl".

Das Verwaltungsgericht wies die Klage ab. Laut Gesetz könne das BfArM den Kauf von Medikamente für einen Suizid nicht erlauben. Ein Recht darauf lasse sich auch aus dem Grundgesetz und der Europäischen Menschenrechtskonvention nicht ableiten.

Gegen diese Entscheidung kann das Paar Berufung beim Oberverwaltungsgericht Nordrhein-Westfalen in Münster einlegen. 2012 hatte der Europäische Gerichtshof für Menschenrechte betont, dass sich die deutschen Gerichte mit der Frage eines Anspruchs auf passive Sterbehilfe beschäftigen müssen. Inhaltlich hatten

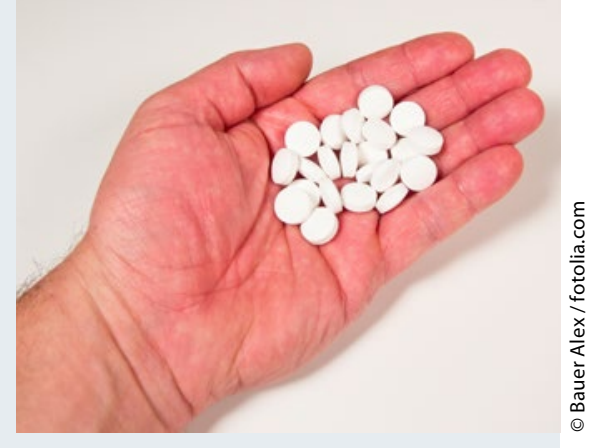

sich die Straßburger Richter dazu aber nicht geäußert. Sie verwiesen allerdings darauf, dass nur vier von 42 untersuchten Zeichnerstaaten der europäischen Menschenrechtskonvention es erlauben, schwer kranken Menschen ein tödliches Medikament für die Selbsttötung zu verschreiben.

Martin Wortmann

Verwaltungsgericht Köln, Az. 7 K 14/15 